\title{
Evaluation of synchronization protocols and methods of early pregnancy diagnosis in dairy cattle
}

\author{
A.H. Shahzad" ${ }^{1 \#}$, A. Sattar ${ }^{1}$, N. Ahmad', I. Ahmad ${ }^{2}$, M.S. Yousaf ${ }^{2}$, S. Abbas ${ }^{2}$, R.S. Blouch ${ }^{3}$, D. \\ $\mathrm{Nak}^{4}$, Y. Nak${ }^{4}$, D.L. Ray ${ }^{5}$ \& W.J. Silvia ${ }^{5}$ (deceased) \\ ${ }^{1}$ Theriogenology Department, University of Veterinary and Animal Sciences, Lahore-Pakistan \\ ${ }^{2}$ Department of Physiology, University of Veterinary and Animal Sciences, Lahore-Pakistan \\ ${ }^{3}$ Livestock and Dairy Development Department, Punjab \\ ${ }^{4}$ Department of Veterinary Obstetrics and, Gynecology Uludag University, Bursa-Turkey \\ ${ }^{5}$ Department of Animal and Food Sciences, University of Kentucky, Lexington 40546-0215
}

(Submitted 15 February 2020; Accepted 3 September 2020; Published 29 November 2020)

\author{
Copyright resides with the authors in terms of the Creative Commons Attribution 4.0 South African Licence. \\ See: http://creativecommons.org/licenses/by/4.0/za \\ Condition of use: The user may copy, distribute, transmit and adapt the work, but must recognise the authors and the South \\ African Journal of Animal Science.
}

\begin{abstract}
The studies aimed to evaluate the pregnancy rate (PR) for timed artificial insemination (TAI) after G7G-Ovsynch, modified G7G-Ovsynch (MG7G-Ovsynch) and Ovsynch protocols and to assess the accuracy of using pregnancy-associated glycoproteins (PAGs) and plasma progesterone $\left(\mathrm{P}_{4}\right)$ in pregnancy diagnosis compared with ultrasonography (US). In study 1 , Holstein cows $(n=37)$ were bred by TAI following the G7G-Ovsynch protocol $(n=19)$ or MG7G-Ovsynch $(n=18)$. Pregnancy was evaluated by US at days 31, 59, and 87 after breeding. The PR was not different for the G7G-Ovsynch and MG7G-Ovsynch. Blood and milk samples were collected on day 3 after insemination and then weekly through day 59 post TAI in cows diagnosed as not pregnant on day 31 and through day 87 in pregnant cows. PAGs were measured using ELISA and $P_{4}$ by radioimmunoassay (RIA). In the second study, Holstein cows $(n=212)$ were bred by TAl following G7G-Ovsynch protocol $(n=110)$ or standard Ovsynch $(n=102)$. Cows were subjected to pregnancy diagnosis on days 30,60 , and 90 . A subset ( $n=15$ in each group) was subjected to blood and milk samples on days $30,45,60,75$, and 90 to measure PAGs and $P_{4}$. In study 2, PR was not significantly different between synchronization protocols on days 30,60, and 90 . Pregnancy loss averaged $15 \%$ between day 30 and day 90 . The use of PAGs and $P_{4}$ proved equally effective in diagnosis of pregnancy. Thus, G7GOvsynch was deemed the protocol of choice in postpartum cows, and PAGs assayed in milk or plasma could be used to diagnose pregnancy.
\end{abstract}

Keywords: artificial insemination, oestrous synchronization, Ovsynch, postpartum cow, pregnancy associated glycoproteins, ultrasonography

${ }^{\#}$ Corresponding author: ahshahzad@uvas.edu.pk

\section{Introduction}

Reproductive efficiency is an important component of overall herd profitability. The factors responsible for efficient reproductive performance include herd health, nutrition, cow comfort, management decisions, manual labour, and improved reproductive management. Accurate heat detection, proper breeding methods and early pregnancy diagnosis (EPD) are instrumental in improving reproductive management. Failure to detect oestrus and improper breeding time, however, are the main constraints to reproductive efficiency. Several reproductive management protocols have been designed for planned breeding and implemented on large commercial dairy setups with mixed results.

Despite increased understanding of reproductive physiology, PR has decreased from $66 \%$ to $28 \%$ over the past five decades (Lucy et al., 2001). Failure to detect oestrus in high-producing dairy cows necessitates TAl-based synchronization strategies. For this purpose, gonadotropin releasing hormone $(\mathrm{GnRH})$-based synchronization protocols are being utilized. These protocols facilitate TAl and eliminate the need for heat detection. A major limitation of these protocols is the lack of tight synchrony of ovulation (Colazo \& Mapletoft, 2014). The introduction of the Ovsynch protocol by Pursley et al. (1995) is believed to be a landmark in controlled breeding. It consists of an injection of $\mathrm{GnRH}$ which causes ovulation 
of a dominant follicle (if present). Seven days later, a $35 \mathrm{mg}$ intramuscular injection of PGF2 $\alpha$ is administered to cause regression of any corpus lutea that is present. Two days later, the cows receive another injection of $\mathrm{GnRH}$ to cause ovulation of the new dominant follicle. Finally, 24 hours later the cows are artificially inseminated. As Ovsynch grew more universal, scientists appraised procedures to fine-tune it. The ovulatory response to the first injection of $\mathrm{GnRH}$ in the Ovsynch protocol was highly variable depending on the time is was administered during the oestrous cycle (Vasconcelos et al., 1999). Based on these findings, many modifications were introduced (Bello et al., 2006; Dirandeh et al., 2015). Presynch protocols consisting of a single injection of $P G$ followed by a GnRH injection two days later, and six or seven days before the standard Ovsynch (G6G or G7G, respectively) improved the PR following TAl (Bello et al., 2006). The G7G-Ovsynch protocol seems to be more practical because most injections are given weekly.

Early pregnancy detection is needed for efficient management of the dairy because non-pregnant animals will need to be either synchronized again or culled. Rectal palpation (RP) and US are commonly used methods for EPD. With RP, pregnancy can be diagnosed at about day 35 post Al by a skilled person (Thompson et al., 2010). Although RP is used widely, and is a simple and low cost method, it may cause untoward pregnancy termination. Ultrasonography is also useful for EPD, and can be used to assess foetal viability and sex. It is less invasive than RP, but requires sophisticated equipment and a degree of technical skill. Elevated $\mathrm{P}_{4}$ concentration in serum or milk between 18 and 24 days post breeding is a valuable indicator of pregnancy, but requires knowing the exact breeding date. Pregnancy associated glycoproteins (PAGs) have been proposed as an alternative bio-marker for pregnancy (Sasser et al., 1986). They can be detected following the third week after breeding in milk and blood.

The main objectives of this study were i) to compare G7G-Ovsynch with MG7G-Ovsynch and standard Ovsynch protocols in postpartum Holstein cows; ii) to evaluate pregnancy status with PAGs, plasma progesterone and US as tools for PD; and iii) to evaluate the effect of factors such as parity, $\mathrm{P}_{4}$, foetal sex and sire effect on PAGs in milk and plasma. It was hypothesized that the G7G-Ovsynch protocol with two injections of $\mathrm{PGF}_{2 \alpha}$ will improve the PR and PAG-based pregnancy diagnosis as an early effective, skilloriented cost-effective tool.

\section{Materials and Methods}

The Institutional Animal Care and Use Committee (IACUC) of the University of Kentucky approved the protocol procedure (IACUC \#2013-1153) of the current study.

The first experiment was conducted with Holstein cows $(n=40)$ maintained at Coldstream Dairy Farm

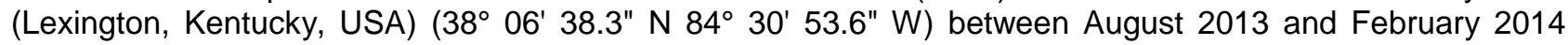
using multiparous (2.5 average lactations) lactating dairy cows. The cows were randomly assigned to two treatments with 20 cows in each. During the study, three cows were removed because of health issues, resulting in the groups having 19 and 18 cows. Cows were housed in free stall barns with one hour access to a pasture daily for exercise. They were fed a total mixed ration (TMR) once daily with ad libitum access to feed and water. Fans were provided in the holding pen and in the free stall barns, and sprinklers were installed above the feed bunk. The cows were milked twice daily (04h30 and 15h30) with the Afimilk (Kibbutz Afikim, Israel) milking system. They were fed a $17 \%$ crude protein-based TMR consisting of corn silage, alfalfa haylage, cottonseed, ground corn, soybean meal, distillers grains, and a mineral mix (with amounts of the ingredients varying slightly throughout the study) at $03 \mathrm{~h} 00$ and $13 \mathrm{~h} 00$. The first group received the G7GOvsynch protocol (day -2 prostaglandin F2 alpha ( $\mathrm{PGF}_{2} \alpha$; Lutalyse, Pfizer, New York, USA), day 0 GnRH (Factrel, Fort Dodge Animal Health, Collegeville, Pennsylvania, USA) day $7 \mathrm{GnRH}$, day $14 \mathrm{PGF}_{2} \alpha$, day 16 $\mathrm{GnRH}$ ) for oestrous synchronization. The second group received a modified treatment (MG7G-Ovsynch) with two injections of PGF2 $\alpha$ administered at eight-hour intervals on day 14. Insemination in both groups was done 20 hours after the final injection of $\mathrm{GnRH}$. All cows were 45 to 90 days post partum at the start of the synchronization protocol and thus 65 to 110 days post partum at the time of Al. Pregnancy was determined using trans-rectal US at day 31 and reconfirmed at days 59 and 87 post Al.

Milk and blood samples were collected from all of the cows, starting three days after insemination and then every seven days. Sampling continued through day 59 post Al if the cow was not diagnosed pregnant on day 31 and through day 87 if the cow was pregnant on day 31 . Milk samples ( $35 \mathrm{~mL}$ ) from each cow were collected in plastic tubes, mixed with bronopol preservative and stored at $4{ }^{\circ} \mathrm{C}$ until they were shipped, for arrival the same day, to IDEXX Laboratories (Westbrook, Maine) to determine PAGs. Blood samples were collected by puncturing the median caudal vein with a syringe, with the blood being drawn into a vacuum tube and centrifuged at 2800x gravity for 20 minutes. After centrifugation, the plasma was transferred to two vials and frozen. One vial was shipped with the milk samples to IDEXX to determine PAG. The other vial was used to quantify $\mathrm{P}_{4}$ using a solid-phase, RIA kit (Coat-a-Count Progesterone, Diagnostic Products Corporation, Los Angeles, CA). The concentration of PAGs in plasma and milk samples was determined with ELISA kit procedures. Both the plasma and milk-based assays were designed to be qualitative, classifying 
the results as pregnant and not pregnant. Reports from IDEXX included the qualitative assessment and optical density (OD).

In the second study, Holstein cows $(n=212)$ maintained at Akbaslar Dairy, Bursa, Turkey, were bred using fixed-time Al (FTAI). The synchronization protocols were G7G-OvSynch, as in the first study, and the conventional Ovsynch protocol. Pregnancy was determined by transrectal US at 30 days after insemination and confirmed 60 and 90 days post Al. Starting on day 30 post Al, only 15 pregnant cows from each treatment were sampled at fortnightly intervals for PAG evaluation in plasma and milk. The procedures were similar to those used in the first study. After milking, the samples were refrigerated and a blood sample (10 $\mathrm{ml}$ ) was collected. The PAG and $\mathrm{P}_{4}$ concentrations were determined with an ELISA kit. Both the plasma and milk-based assays were designed to be qualitative, classifying the cows as pregnant or not pregnant.

The effects of treatments and parity on pregnancy rates were analysed with chi-square procedures in PROC FREQ of SAS (SAS Institute, Inc., Cary, North Carolina, USA). The effects of treatment on PAGs in milk and plasma and $\mathrm{P}_{4}$ profiles in plasma were determined with the GLM procedure of SAS. The accuracy of the milk-based ELISA was determined by comparing the positive predictive values (PPV) and negative predictive values (NPV) of the assay to the established plasma-based assay. The sensitivity of the assay was evaluated by comparing the adjusted OD measurements of samples collected from pregnant and nonpregnant cows over time. Correlations of $\mathrm{P}_{4}$ with the concentrations of PAGs in serum and milk were quantified using the REG procedure of SAS. Effects were declared significant at $P<0.05$ and a trend towards significance was indicated when $P \leq 0.10$.

\section{Results and Discussion}

In the first study, the PR in the cows of the G7G-Ovsynch group was $47 \%$ compared with $50 \%$ in the MG7G-Ovsynch group at day 31 post $\mathrm{Al}(P=0.87)$. At days 59 and 87 post $\mathrm{Al}$, PR was recorded as $37 \%$ on both days in the G7G-Ovsynch group, compared with slightly lower values (33\%) in MG7G-Ovsynch $(P$ $=0.82$ ). Overall pregnancy loss in the G7G-Ovsynch group was $11 \%$, whereas in MG7G-Ovsynch it was $17 \%$ $(P=0.59)$ (Table 1). In the second study, PR in the G7G-Ovsynch group was non-significantly $(P=0.16)$ higher $(52 \%)$ compared with those of the Ovsynch group (42\%) on day 30 post Al. On day 60 post Al, PR was $45 \%$ in the G7G-Ovsynch group compared with the Ovsynch group, in which PR was $37 \%(P=0.23)$. Pregnancy date on day 90 was $44 \%$ in the G7G-Ovsynch group in comparison with $36 \%$ in the Ovsynch group $(P=0.28)$. Overall pregnancy loss in the $\mathrm{G} 7 \mathrm{G}$-Ovsynch group was $16 \%$ and in the Ovsynch group it was $14 \%(P=0.80)($ Table 2$)$.

Table 1 Pregnancy rates and losses in lactating dairy cows synchronized and inseminated by timed artificial insemination

\begin{tabular}{lrrrrrr}
\hline & \multicolumn{3}{c}{ First experiment } & \multicolumn{3}{c}{ Second experiment } \\
\cline { 2 - 7 } Variable $^{1}$ & \multicolumn{1}{c}{ G7G } & MG7G & $P$-value & G7G & Ovsynch & $P$-value \\
\hline Pregnancy rate, 31 (30) days post Al & $47 \%(9 / 19)$ & $50 \%(9 / 18)$ & 0.87 & $52 \%(57 / 110)$ & $42 \%(43 / 102)$ & 0.16 \\
Pregnancy rate, 59 (60) days post Al & $37 \%(7 / 19)$ & $33 \%(6 / 18)$ & 0.82 & $45 \%(50 / 110)$ & $37 \%(38 / 102)$ & 0.23 \\
Pregnancy rate, 87 (90) days post Al & $37 \%(7 / 19)$ & $33 \%(6 / 18)$ & 0.82 & $44 \%(48 / 110)$ & $36 \%(37 / 102)$ & 0.28 \\
Pregnancy loss, 59 (60) days post Al & $11 \%(2 / 19)$ & $17 \%(3 / 18)$ & 0.59 & $12 \%(7 / 57)$ & $12 \%(5 / 43)$ & 0.92 \\
Pregnancy loss, 87 (90) days post Al & $0 \%(0 / 19)$ & $0 \%(0 / 18)$ & ---- & $4 \%(2 / 50)$ & $3 . \%(1 / 38)$ & 0.72 \\
$\begin{array}{l}\text { Pregnancy loss, 31 (30) to 87 (60) } \\
\text { days post Al }\end{array}$ & $11 \%(2 / 19)$ & $17 \%(3 / 18)$ & 0.59 & $16 \%(9 / 57)$ & $14 \%(6 / 43)$ & 0.80 \\
\hline
\end{tabular}

${ }^{1}$ Number of days given in parentheses is for the second experiment

Al: artificial insemination; TAl: timed artificial insemination

The animals that had been used in the first study were divided into two groups by parity. One group contained animals in their first and second parities $(n=23)$ and the second group contained animals in their third to fifth parities $(n=14)$. Pregnancy rates were significantly different between these groups (Table 2). Four pregnancies (57\%) were lost between day 31 and day 59 in the cows in their first or second parity. 
Table 2 Effects of parity on pregnancy rates following oestrous synchronization and timed artificial insemination

\begin{tabular}{lccc}
\hline Rates & 1st and 2nd parity & 3rd to 5th parity & $P$-value \\
\hline Pregnancy 31 days post Al & $30 \%(7 / 23)$ & $71 \%(10 / 14)$ & 0.02 \\
Pregnancy 59 days post Al & $13 \%(3 / 23)$ & $71 \%(10 / 14)$ & $<0.01$ \\
Pregnancy 87 days post Al & $13 \%(3 / 23)$ & $71 \%(10 / 14)$ & $<0.01$ \\
Calving rate & $13 \%(3 / 23)$ & $71 \%(10 / 14)$ & $<0.01$ \\
\hline
\end{tabular}

In the first study, pregnancy was diagnosed with ELISA-based assays of PAGs from milk and plasma, plasma $\mathrm{P}_{4}$ and trans-rectal US on days $31,59,87$. The results of pregnancy diagnoses using milk and plasma PAGs and plasma $\mathrm{P}_{4}$ were compared for those animals that were diagnosed pregnant by US (Table 3). Overall, 18 animals were pregnant on day 31 after Al. These results were considered 'gold standard' and compared with milk- and plasma-based PAGs and $\mathrm{P}_{4}$ on days 31,59 , and 87 post FTAI (Table 4). In the ELISA-based PAG method, sensitivity was $100 \%$ (range $92 \%$ to $100 \%)(P<0.05)$. For the same method, specificity was $56 \%(P<0.05)$ with a range of 21 to $86(P<0.05)$. The positive predictive value (PPV) was $92 \%(80 \%$ to $98 \%)(P<0.05)$. The negative predictive value (NPV) for the ELISA-based pregnancy method was $100 \%(48 \%$ to $100 \%)(P<0.05)$. The sensitivity of ELISA-based plasma PAGs $(\mathrm{n}=$ $54)$ was recorded as $98 \%(88 \%$ to $100 \%)$ and specificity was $56 \%$. Positive predictive value was $92 \%(80$ 98\%) $(P<0.05)$. Negative predictive value was as $83 \%(36-100 \%)(P<0.05)$ which was comparable with milk-based PAGs method. The third PD was carried out by measuring the $\mathrm{P}_{4}$ concentration with RIA. The sensitivity of this method was $98 \%(88-100 \%)(P<0.05)$ and sensitivity was $89 \%$. The positive predictive value $(\mathrm{PPV})$ was $98 \%(88-100 \%)(P<0.05)$ and NPV was $89 \%(52-100 \%)(P<0.05)$.

Table 3 Optical density from the assay of pregnancy associated glycoproteins in milk and plasma and progesterone concentration in postpartum, pregnant dairy cows

\begin{tabular}{lrcc}
\hline Diagnosis & PAGs in milk, OD & PAGs in plasma, OD & $\mathrm{P}_{4}$ in plasma, $\mathrm{ng} / \mathrm{mL}$ \\
\hline True pregnant & 45 & 44 & 44 \\
False pregnant & 4 & 4 & 1 \\
True non-pregnant & 5 & 5 & 8 \\
False non-pregnant & 0 & 1 & 1 \\
Assay characteristics & & & \\
Sensitivity & 100 & 98 & 98 \\
Specificity & 56 & 56 & 89 \\
Positive predictive value & 92 & 92 & 98 \\
Negative predictive value & 100 & 83 & 89
\end{tabular}

${ }^{1}$ Sensitivity: percentage of animals diagnosed as pregnant that were truly pregnant; specificity: percentage of animals diagnosed as not pregnant that were truly not pregnant; positive predictive value: ability to correctly identify pregnant animals, negative predictive value: ability to correctly identify non pregnant animals

PAGs: pregnancy-associated glycoproteins, $\mathrm{P}_{4}$ : progesterone. OD: optical density

Foetal sex has been shown to affect the PAG profile in the peri-partum period (Gabor et al., 2007). However, Serrano et al. (2009) and López-Gatius et al. (2007) found no such effects from days 40 to 210 of pregnancy. In the present study, no effect was observed of the sex or the sire of the foetus on the profile of milk or plasma PAGs (Figure 1).

The $\mathrm{P}_{4}$ level in serum was positively correlated with the concentration of PAGs in milk and in plasma $(r$ $=0.46$ and 0.42 , respectively. The levels of PAGS in milk and plasma were also highly correlated $(r=0.85)$. 

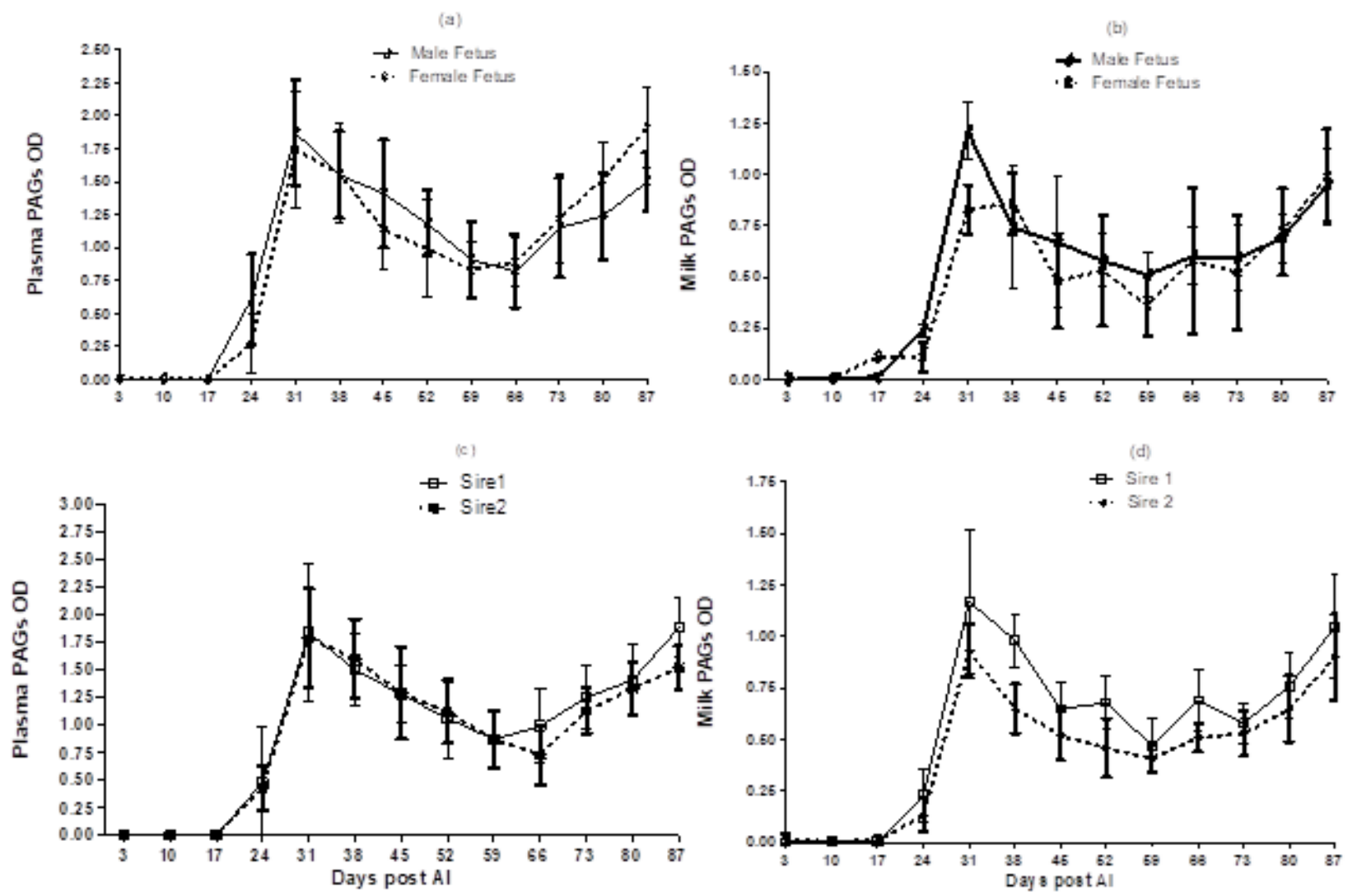

Figure 1 Effect of foetal sex on (a): plasma PAG profile (optical density, OD) and (b): Milk PAGs profile; effect of sires on (c): Plasma PAGs profile and (d): Milk PAGs profile on weekly basis in Holstein postpartum dairy cows during first trimester of gestation period

In Study 2, the difference between synchronization protocols was not significant $(P<0.05)$ for OD of the plasma PAGs on days 30, 45 and 75 post Al, but was significant on days $60(P<0.01)$ and $90(P=0.01)$ (Table 4). The difference between synchronization protocols in optical density of PAGs measured in milk was not significant except on day $75(P=0.02)$ post Al. The difference in synchronization protocols did not affect $\mathrm{P}_{4}$ levels at any point.

Table 4 Pregnancy associated glycoproteins in plasma and milk and the plasma concentration of progesterone on days 30, 45, 60, 75 and 90 post Al in lactating dairy cows synchronized by G7G-Ovsynch and Ovsynch protocols in Study 2

\begin{tabular}{lcccccc}
\hline \multirow{2}{*}{$\begin{array}{l}\text { Days } \\
\text { post Al }\end{array}$} & \multicolumn{3}{c}{ G7G-Ovsynch } & & \multicolumn{2}{c}{ Ovsynch } \\
\cline { 2 - 7 } & PAGs in plasma & PAGs in milk & $P_{4}$ & PAGs in plasma & PAGs in Milk & $P_{4}$ \\
\hline 30 & $0.85 \pm 0.12$ & $1.43 \pm 0.12$ & $5.84 \pm 0.23$ & $0.75 \pm 0.08$ & $1.54 \pm 0.12$ & $5.74 \pm 0.35$ \\
45 & $0.56 \pm 0.08$ & $1.35 \pm 0.06$ & $6.47 \pm 0.20$ & $0.45 \pm 0.05$ & $1.23 \pm 0.12$ & $6.66 \pm 0.32$ \\
60 & $0.59 \pm 0.06$ & $1.18 \pm 0.09$ & $7.90 \pm 0.22$ & $0.35 \pm 0.04$ & $1.10 \pm 0.12$ & $7.73 \pm 0.22$ \\
75 & $0.62 \pm 0.15$ & $1.36 \pm 0.09$ & $7.74 \pm 0.30$ & $0.50 \pm 0.04$ & $1.07 \pm 0.06$ & $7.60 \pm 0.41$ \\
90 & $0.86 \pm 0.08$ & $1.66 \pm 0.08$ & $8.47 \pm 0.27$ & $0.61 \pm 0.04$ & $1.74 \pm 0.08$ & $8.29 \pm 0.30$ \\
\hline
\end{tabular}

PAGs: pregnancy associated glycoproteins, $\mathrm{P}_{4}$ : progesterone 
The results of the present studies are in accord with the findings of Dirandeh et al. (2015), who used an initial injection of prostaglandin or $\mathrm{GnRH}$ to induce ovulation, followed by $\mathrm{GnRH}$ six or seven days later, with the use of prostaglandin being more effective. The recorded PR was approximately $41 \%$ on day 32 post $\mathrm{Al}$ and approximately $35 \%$ on day 60 . Thus, $6 \%$ of the pregnancies were lost between day 32 and day 60 . Based on these results, Dirandeh et al. (2015) concluded that G7G-Ovsynch, which was practical in execution, could be used with confidence as most injections were given on the same day of the week. In the present study, the rates of pregnancy and pregnancy loss were slightly greater at comparable times than in Dirandeh et al. (2015).

A non-invasive tool to detect pregnancy early in gestation is an important factor in optimal reproductive management. During the last three decades PAGs have proved a suitable indicator of pregnancy for use early in gestation (Sasser et al., 1986). Besides being an indicator of pregnancy, the PAG profile is useful in assessing placental competency and the successful continuation of pregnancy. An atypical PAG profile may indicate embryonic mortality and loss of a pregnancy (Butler et al., 1982; Friedrich \& Holtz 2010; LópezGatius et al., 2007; Pohler et al., 2013). Pohler et al. (2013) documented overall 19\% LEM. Approximately $50 \%$ of late embryonic mortality occurs between days 28 and 42 post Al (Silke et al., 2002) and results in a prolonged post-partum interval to rebreeding. From a managerial point of view, accurate pregnancy diagnosis is essential in minimizing late embryonic mortaility (Pohler et al., 2013). These authors observed that on day 31 post Al, PAGs were a reasonable indicator of pregnancy and might provide insight into the successful maintenance of pregnancy beyond this point.

There was a positive correlation between $\mathrm{P}_{4}$ profile and milk or plasma PAGs in the present study. Pregnancy-associated glycoproteins appear to regulate luteal secretion of $P_{4}$ from days 50 to 90 of pregnancy through the stimulation of prostaglandin E secretion (Weems et al., 2007). In the present study, PAGs were detectable in the third week of gestation, increased through the fourth week, and then decreased. After this declining phase, there was a gradual rise in PAGs until the last day of sampling in the present study.

High sensitivity is an essential feature of any EPD test. In the present study, the percentage of animals diagnosed as pregnant that were truly pregnant was $100 \%$ and $98 \%$ in tests that used milk and plasma. Thus, these tests were highly sensitive. However, the percentage was higher of animals diagnosed as not pregnant that were truly not pregnant. Therefore, the specificity of these pregnancy tests was lower. Truly pregnant animals that were diagnosed as not being pregnant might be subjected to another round of hormonal treatment, which could result in the loss of their pregnancy and a prolonged intercalving period. In the same way, low specificity leads to misdiagnosis of cows as pregnant when they should be in the true non-pregnant category according to their real pregnancy status, which also lengthens their intercalving period. Like the present results, Giordano et al. (2013) found the sensitivity of EPD with PAGs 1.8 times greater than the specificity of the test. Any test that has an error rate that is equal to or less than $3 \%$ is deemed acceptable as a tool for PD. Ferguson and Galligan (2011) considered that any test that had less than $90 \%$ sensitivity was not viable. Giordano et al. (2013) proposed stricter criteria with the suggestion that sensitivity be greater than $96 \%$ on day $31 \pm 3$ and greater than $94 \%$ on day $24 \pm 3$ post Al.

\section{Conclusion}

Pregnancy-associated glycoproteins in milk and plasma were at least as accurate as ultrasonography in identifying pregnant and non-pregnant Holstein cows at the end of the first month of gestation. Thus, PAGbased tests could serve as a viable alternative for EPD by dairy farmers who are unable to obtain veterinary assistance. The G7G-Ovsynch protocol should be evaluated in animals with more than three parities. Given the small number of observations, no definitive conclusion could be drawn from the observed $6 \%$ difference between synchronization protocols in the PR of cows in their first lactation. However, the observed numerical advantage in PR with the G7G-Ovsynch protocol may make it the protocol of choice in postpartum cows.

\section{Acknowledgements}

Funding is acknowledged received from the Higher Education Commission (HEC), Islamabad under 5000 indigenous fellowships for experiment 1 and TUBITAK for execution of experiment 2.

\section{Author Contributions}

AHS and DLR executed the project under the supervision of WJS in study 1. YN and DN executed, analysed and interpreted the data in study 2. AHS collected blood samples and compiled the data. AS supervised compilation of data. WJS and YN conceived and planned experiments. IA, RSB, NA, AHS, MSY and SA analysed and interpreted the data, wrote the manuscript and prepared the figures.

\section{Conflict of Interest Declaration}

The authors have no conflict of interest to declare. 


\section{References}

Bello, N. M., Steibel, J.P. \& Pursley, J.R., 2006. Optimizing ovulation to first GnRH improved outcomes to each hormonal injection of Ovsynch in lactating dairy cows. J. Dairy Sci. 89, 3413-3424.

Butler, J.E., Hamilton, W.C., Sasser, R.G., Ruder, C.A., Haas, G.M. \& Williams, R.J., 1982. Detection and partial characterization of two bovine pregnancy-specific proteins. Biol. Reprod. 26, 925-933.

Colazo, M. G. \& Mapletoft, R. J., 2014. A review of current timed AI (TAI) programs for beef and dairy cattle. Can. Vet. J. $55,772-780$.

Dirandeh, E., Rezaei, A. \& Colazo, M. G.2015. Double-Ovsynch, compared with Presynch with or without GnRH, improves fertility in heat-stressed lactating dairy cows. Theriogenology $83,438-443$

Ferguson, J.D. \& Galligan, D.T., 2011. The value of pregnancy diagnosis - A revisit to an old art. Theriogenology Annu. Conf. Symp. Milwaukee, WI.

Friedrich, M. \& Holtz, W., 2010. Establishment of an ELISA for measuring bovine pregnancy-associated glycoprotein in serum or milk and its application for early pregnancy detection. Reprod. Domest. Anim. 45, 142-146.

Gabor, G.F., Toth, L., Ozsvari, Z., Abonyi-Toth, Z. \& Sasser, R.G., 2007. Early detection of pregnancy and embryonic loss in dairy cattle by ELISA tests. Reprod. Domest. Anim. 42, 633-636.

Giordano, G.O., Fricke, P.M. \& Cabrera, V.E., 2013. Economics of resynchronization strategies including chemical tests to identify nonpregnant cows. J. Dairy Sci. 96, 949-961.

López-Gatius, F., Garbayo, J.M., Santolaria, P., Yániz, J., Ayad, A., de Sousa, N.M., \& Beckers, J.F., 2007. Milk production correlates negatively with plasma levels of pregnancy associated glycoprotein (PAG) during the early fetal period in high producing dairy cows with live fetuses. Domest. Anim. Endocrinol. 32, 29-42.

Lucy, M.C., Billings, H.J., Butler, W.R., Ehnis, L.R., Fields, M.J., Kesler, D.J., Kinder, J.E., Mattos, R.C., Short, R.E., Thatcher, W.W., Wettemann, R.P., Yelich, J.V. \& Hafs, H.D., 2001. Efficacy of an intravaginal progesterone insert and an injection of PGF2 $\alpha$ for synchronizing estrus and shortening the interval to pregnancy in postpartum beef cows, peripubertal beef heifers, and dairy heifers. J. Anim. Sci. 79, 982-995.

Pohler, K.G., Geary, T.W., Johnson, C.L., Atkins, J.A., Jinks, E.M., Busch, D.C., Green, J.A., MacNeil, M.D. \& Smith, M.F., 2013. Circulating bovine pregnancy associated glycoproteins are associated with late embryonic/fetal survival but not ovulatory follicle size in suckled beef cows. J. Anim. Sci. 91, 4158-4167.

Pursley, J.R., Mee, M.O, \& Wiltbank, M.C., 1995. Synchronization of ovulation in dairy cows using PGF2a and GnRH. Theriogenology 44,915-923.

Sasser, R.G., Ruder, C.A., Ivani, K.A. \& Butler, J.A., 1986. Hamilton WC. Detection of pregnancy by radioimmunoassay of a novel pregnancy-specific protein in serum of cows and a profile of serum concentrations during gestation. Biol. Reprod. 35, 936-942.

Serrano, B., López-Gatius, F., Santolaria, P., Almería, S., García-Ispierto, I., Bech-Sabat G., Sulon, J., de Sousa, N.M., Beckers, J.F. \& Yániz, J.L., 2009. Factors affecting plasma pregnancy-associated glycoprotein-1 concentrations throughout gestation in high-producing dairy cows. Reprod. Domest. Anim. 44, 600-605.

Silke, V., Diskin, M.G,, Kenny, D.A., Boland, M.P., Dillon, P., Mee, J.F. \& Sreenan, J.M., 2002. Extent, pattern and factors associated with late embryonic loss in dairy cows. Anim Reprod Sci. 71: 1-12.

Thompson, I.M., Derri, R.L., Kim, I.H., Green, J.A., Santos, J.E. \& Thatcher, W.W., 2010. Effects of resynchronization programs on pregnancy per artificial insemination, progesterone, and pregnancy-associated glycoproteins in plasma of lactating dairy cows. J. Dairy Sci. 93, 4006-4018.

Vasconcelos, J.L.M., Silcox, R.W., Rosa, G.J.M., Pursley, J.R. \& Wiltbank, M.C., 1999. Synchronization rate, size of the ovulatory follicle, and pregnancy rate after synchronization of ovulation beginning on different days of the estrous cycle in lactating dairy cows. Theriogenology 52, 1067-78.

Weems, Y.S., Kim, L., Humphreys, V., Tsuda, V., Blankfein, R., Wong, A. \& Weems, C.W., 2007. Effect of luteinizing hormone (LH), pregnancy-specific protein $B(P S P B)$, or arachidonic acid (AA) on secretion of progesterone and prostaglandins (PG) E (PGE; PGE(1) and PGE(2) and F(2alpha) (PGF(2alpha)) by ovine corpora lutea of the estrous cycle or pregnancy in vitro. Prostaglandins Other Lipid Mediat. 84,163-173. 